\title{
Evaluation of overall survival (os) in patient with chronic lymphocytic leukemia in West of Iran in 1998-2016
}

\begin{abstract}
Background \& Aim: Chronic lymphocytic leukemia (CLL) is the most common lymphoid leukemia in Western Countries. Also, in Asian countries, such as Iran is expanding. This study was investigated in Kermanshah province in 73 patients newly diagnosed with chronic lymphocytic leukemia, and the aim of this paper is the evaluation of overall survival (OS) rate and the factors affecting it.
\end{abstract}

Methods and material: This is a descriptive-cross sectional study on 73 patients with CLL during 1998-2016 and patients were classified according to clinical symptoms, age's mean, sex, laboratory findings, diagnostic tests, the therapeutic regimens and side effects from them. Data were entered into SPSS, CAN-Reg4 and all tests were performed at the $5 \%$ level of significance using SPSS version 17.0. Patients with CLL have referred to related clinical oncologist have been evaluated in our paper from Kermanshah province during 18 years.

Results: According to our findings from this study the mean age of the patients at diagnosis was 59.58 years that $53.4 \%$ were male. Mean of OS in patients was 175.53 months, in a detail, mean of survival in 39 male patients was 128.52 months and in 34 female patients was 190.66 months. Also, OS was associated with the stage of disease (CLL), sex and treatment. The 5-year survival and the ten-year survival rate of patients were $89 \%$ and $81 \%$, respectively.

Conclusion: According to recently progression in CLL treatment, but this malignancy still incurable and this is an important point for the evaluation of survival rate in this patients. Based on our findings in addition to significantly increasing in OS, related factors also are important and efficient on OS.

Keyword: chronic, lymphocytic, leukemia, survival
Volume 9 Issue I - 2018

\begin{abstract}
Afshin Karami,' Noorodin Karami, ${ }^{2}$ Mehrdad Payandeh, ${ }^{3}$ Amir Yami, 'Sahel Kahrizi ${ }^{4}$

'Department of Hematology, Shahid Beheshti University of Medical Sciences, Iran

${ }^{2}$ Department of Genetics, Shahid Sadoughi University of Medical Science, Iran

${ }^{3}$ Department of Hematology and Medical Oncology, Kermanshah University of Medical Sciences, Iran

${ }^{4}$ Department of Laboratory Sciences, Kermanshah University of Medical Sciences, Iran
\end{abstract}

Correspondence: Mehrdad Payandeh, Department of Hematology and Medical Oncology, University of Medical Sciences, Kermanshah, Iran, Email afshinkarami72@gmail.com

Received: October 28, 2017| Published: January 23, 2018

\section{Introduction}

Chronic lymphocytic leukemia (CLL) is a type of lymphoid neoplasms that occurs with the accumulation of clonal b-cell lymphocytes in organs such as bone marrow, blood and lymph nodes. ${ }^{1}$ This leukemia is one of the most common leukemia in western countries which appear in adults, however; the prevalence of CLL in eastern countries is lower than western countries. ${ }^{2,3}$ However, this malignancy includes about 19,000 cases of all newly diagnosed cases of cancer in the United States in $2016 .{ }^{4}$ One of the most important pathophysiological causes in CLL patients is genetic variation that is very widespread, as well as chromosomal alterations in at least $80 \%$ of these patients in one of the following forms. Chromosomal alterations: a deletion in chromosome 13q14.3 (del 13q), del (11q), del (17p) and trisomy $12.5,6$

Treatment of this leukemia is based on clinical symptoms, in other words, the patients without pathologic sings are free from treatment while the patients with signs are needed for the cure and this highlights the importance of prognosis of this disorder.,

1. Survival of a patient with CLL is nearly $80 \%$ but lots of this statistics have belonged to western countries. There is a lot of paper that allocated their focus to survival in this disorder e.g.

2. The article about compare of survival in Germany with America in $2016 .^{9}$
3. Leukemia monitoring and survival evaluation and related factors like age and sex in 2008 in Germany. ${ }^{10,11}$

4. Improving in CLL treatment in Taiwan during 1990-2004. ${ }^{12}$

5. In this study, we evaluate the overall survival of the patients with CLL in the west of Iran, Kermanshah during 18 years for the first time.

\section{Materials and methods}

This is a retrospective and descriptive cross-sectional study on 73 newly diagnosed patients in the west of Iran, Kermanshah province. We have been evaluated overall survival in this patient and factors which have influences on survival.

All information presented in this paper are gotten from the 19982016 from the clinic of hematology-oncology with consent from patients. Age's mean, sex, clinical and laboratory findings, indicative tests, the regimen of medicines and side effects have been performed. $53.4 \%(n=39)$ of these patients were male and $46.6 \%(n=34)$ were female.

We have been used this software for analyzed data: SPSS and Graph-pad.5-year and 10-year overall survival in both of sex have been analyzed and also the influence of epidemiological factors with Cox regression model in SPSS have been evaluated. The Statistical significance was defined by the $\mathrm{p}<0.05$. 


\section{Results}

The mean of patients in this study was 59.47-year and overall survival's mean of these patients was 175.53 months. These tests have been performed for patients due to diagnosing CLL Table 1 .

Also, the average of complete blood count (CBC) in CLL patients was as follows Table 2 .

The patients are classified into two staging systems as follows Tables $3 \& 4$.

Table I Tests have been performed for patients due to diagnosing CLL

\begin{tabular}{llll}
\hline Tests & Flow Cytometry & LDH & Cytogenetic \\
\hline $\mathrm{N}$ & $27^{*}$ & 52 & $2^{*}$ \\
\hline
\end{tabular}

*The tests flow cytometry was included evaluation CD5, CD I9, CD 20, CD23, CD45, CD22 and Ag Ki67.

*2 patients have been performed cytogenetic tests (due to economic causes) and they had a Del.I $3 q 14$.

Table 2 Average of complete blood count (CBC) in CLL patients.

\begin{tabular}{lll}
\hline Variable & Mean & Range \\
\hline WBC $\times 103 / \mu \mathrm{L}$ & 50.9 & $3.1-265$ \\
$\mathrm{Hb} g / \mathrm{dL}$ & 12.3 & $3.9-18.1$ \\
$\mathrm{Plt} \times 103 / \mu \mathrm{L}$ & 175.5 & $30-540$ \\
\hline
\end{tabular}

Table 3 \& 4 Patients are classified into two staging systems that is Ra classification \& Binet classification

\begin{tabular}{ll}
\hline Stage & $\mathbf{N}$ \\
\hline 0 & 16 \\
1 & 19 \\
2 & 18 \\
3 & 11 \\
4 & 9 \\
\hline Stage & $\mathbf{N}$ \\
\hline A & 35 \\
B & 18 \\
C & 20 \\
\hline
\end{tabular}

According to our extracted data from SPSS and other software, the 5 -year and 10-year survival in male and female is presented in below Figure 1.

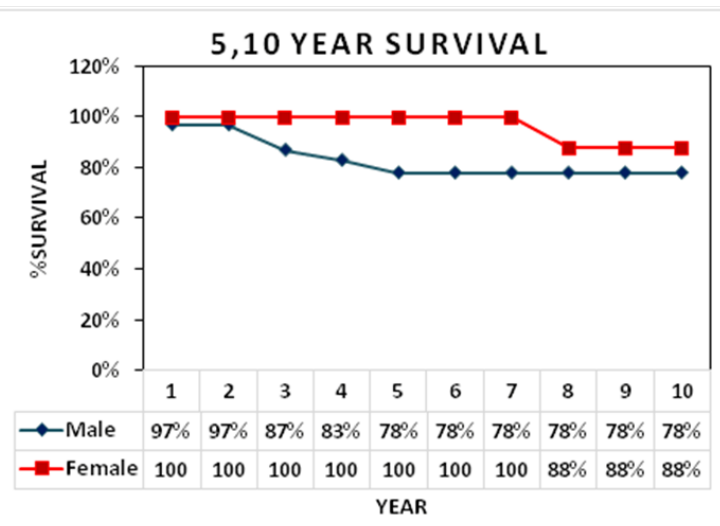

Figure I 5-year and 10-year survival in male and female.

The 5-year and 10-year survival in females are altogether higher than OS in males and this pattern demonstrates the important influence of sex factor on survival. Additionally, we've been found a noteworthy relation between treatment and sex and stage of disease with overall survival's means, $\mathrm{P}=0,006$ and 0.049 and 0.002 respectively.

\section{Discussion}

\section{Treatment protocol}

Treatment protocols for chronic lymphocytic leukemia (CLL) are given below, including the following:

1. Treatment for symptomatic disease:

2. Different single-agent and blend regimens

3. Special considerations for treatment

4. Response assessmentTreatment recommendations for symptomatic CLL:

Fludarabine-based therapy (FCR) - Chlorambucil therapy - Pentostatin-based therapy - Bendamustine-based therapy Alemtuzumab therapy - Ibrutinib therapy -Lenalidomide therapy. ${ }^{13}$

During the treatment process, 57 patients from all with the first line treatment with chlorambucil and 12 patients with combination chemotherapy regimen (CHOP-CVAD-FC-FCR) and 3 patients with fludarabine that are presented in below Table 5 .

Table 5 Treatment recommendations for symptomatic CLL

\begin{tabular}{llll}
\hline Treatment & Chlorambucil & Fludarabine & $\begin{array}{l}\text { Chemotherapy } \\
\text { Regimen }\end{array}$ \\
\hline $\mathrm{N}$ & 57 & 3 & 12 \\
Mean of Survival & 185.8 month & 56.5 month & 74.3 month \\
\hline
\end{tabular}

This table demonstrates that first-line treatment with chlorambucil has the best effect on the survival of patients, also during the treatment process, 8 patients died and 2 patients were affected by Richter's syndrome.

According to information obtained in the analysis, only 43 patients were affected by hematological side effects and the common of a side effect was thrombocytopenia. The side effects of these treatments are presented in below Table 6 .

Table 6 Side effects of the treatments are presented

\begin{tabular}{lllll}
\hline $\begin{array}{l}\text { Side } \\
\text { Effects }\end{array}$ & Anemia & Leukopenia & Thrombocytopenia & Polycythemia \\
\hline $\mathrm{N}$ & 10 & 10 & 26 & 2 \\
\hline
\end{tabular}

Infection is one of the main causes of death in CLL. Enhanced treatment effectiveness and decreased immune toxicity of treatment could have changed the range and outcomes of infections in patients with CLL. ${ }^{14}$ In this study, only 18 patients have got an infection due to vaccination management.

Finally, enhancements in supportive care for patients with CLL probably contributed considerably to the enhanced general survival for all them. Here, enhanced administrations of infections and immune system cytopenia alongside better support for transfusion of blood items and additionally immunoglobulin substitution may have had a huge impact. ${ }^{15}$

\section{Acknowledgements}

None.

\section{Conflicts of interest}

The authors declare that they have no conflict of interests. 


\section{Funding}

None.

\section{References}

1. Rombout A, Verhasselt B, Philippé J. Lipoprotein lipase in chronic lymphocytic leukemia: function and prognostic implications. European Journal of Haematology. 2016;97(5):409-415.

2. Dores GM, Anderson WF, Curtis RE, et al. Chronic lymphocytic leukaemia and small lymphocytic lymphoma: overview of the descriptive epidemiology. Br J Haematol. 2007;139(5):809-819.

3. Payandeh M, Sadeghi M, Sadeghi E. Cholorambucil versus Cholorambucil Plus Prednisolone as First-Line Therapy of Chronic Lymphocytic Leukemia in West of Iran. Iran $J$ Cancer Prev. 2015;8(2):94-99.

4. Kipps TJ, Stevenson FK, Wu CJ, et al. Chronic lymphocytic leukaemia Nat Rev Dis Primers. 2017;3:16096.

5. Lai YY, Huang XJ. Cytogenetic characteristics of B cell chronic lymphocytic leukemia in 275 Chinese patients by fluorescence in situ hybridization: a multicenter study. Chin Med $J$ (Engl). 2011;124(16):2417-2422.

6. Döhner H, Stilgenbauer S, Benner A, et al. Genomic aberrations and survival in chronic lymphocytic leukemia. $N$ Engl J Med. 2000;343(26):1910-1916.

7. Montserrat E, Rozman C. Chronic lymphocytic leukaemia treatment. Blood reviews. 1993;7(3):164-175.
8. Eichhorst B, Robak T, Montserrat E, et al. Chronic lymphocytic leukaemia: ESMO Clinical Practice Guidelines for diagnosis, treatment and follow-up. Ann Oncol. 2015;26(suppl 5):v78-v84.

9. Pulte D, Castro FA, Jansen L, et al. Trends in survival of chronic lymphocytic leukemia patients in Germany and the USA in the first decade of the twenty-first century. J Hematol Oncol. 2016;9(1):28.

10. Nennecke A, Wienecke A, Kraywinkel K. Leukemia incidence and survival in Germany according to current standardized categories. Bundesgesundheitsblatt, Gesundheitsforschung, Gesundheitsschutz. 2014;57(1):93-102.

11. E Hiripi A, Gondos K, Emrich B, et al. Survival from common and rare cancers in Germany in the early 21st century. Annals of Oncology. 2011;23(2):472-479.

12. Wu SJ, Chiang CJ, Lin CT, et al. Improving but inferior survival in patients with chronic lymphocytic leukemia in Taiwan: a populationbased study, 1990-2004. PLoS One. 2013;8(4):e62930.

13. Sandy D Kotiah. Chronic Lymphocytic Leukemia Treatment Protocols. Hematology and Medical Oncology, Mercy Medical Center. 2016.

14. Annalynn M Williams, Andrea M Baran, Philip J Meacham, et al. High Risk of Infections in Chronic Lymphocytic Leukemia Patients Treated with B-Cell Receptor Inhibitors. Blood. 2016;128:3203.

15. da Cunha-Bang C, Simonsen J, Rostgaard K, et al. Improved survival for patients diagnosed with chronic lymphocytic leukemia in the era of chemo-immunotherapy: a Danish population-based study of 10455 patients. Blood Cancer J. 2016;6(11):e499. 